\title{
BMJ Open School-based lifestyle education involving parents for reducing subjective psychosomatic symptoms in Japanese adolescents: study protocol for a cluster randomised controlled trial
}

\author{
Junko Watanabe, ${ }^{1}$ Mariko Watanabe, ${ }^{2,3}$ Kazue Yamaoka, ${ }^{4}$ Misa Adachi, ${ }^{4,5}$ \\ Asuka Nemoto, ${ }^{4}$ Toshiro Tango ${ }^{4,6}$
}

To cite: Watanabe J,

Watanabe M, Yamaoka K, et al. School-based lifestyle education involving parents for reducing subjective psychosomatic symptoms in Japanese adolescents: study protocol for a cluster randomised controlled trial. BMJ Open 2018;8:e018938. doi:10.1136/ bmjopen-2017-018938

- Prepublication history and additional material for this paper are available online. To view these files, please visit the journal online (http://dx. doi org/10.1136/bmjopen-2017018938).

Received 1 August 2017 Revised 1 December 2017 Accepted 12 December 2017

\section{Check for updates}

${ }^{1}$ The Department of Nutrition Management, Minami Kyushu University, Miyazaki, Japan ${ }^{2}$ Showa Women's University, Tokyo, Japan

${ }^{3}$ Prefectural University of Kumamoto, Kumamoto, Japan

${ }^{4}$ Teikyo University Graduate School of Public Health, Tokyo, Japan

${ }^{5}$ Nutrition Support Network LLC, Sagamihara, Japan

${ }^{6}$ Center for Medical Statistics, Tokyo, Japan

Correspondence to Professor Kazue Yamaoka; kazue@med.teikyo-u.ac.jp

\section{ABSTRACT}

Introduction Severe subjective psychosomatic symptoms (SPS) in adolescents are a major public health concern, and lifestyle modification interventions for reducing SPS are important topics. Recently, we developed a schoolbased lifestyle education involving parents for reducing SPS of adolescents (SPRAT), an improved version of the programme from our previous study Programme for adolescent of lifestyle education in Kumamoto (PADOK). This study aimed to evaluate the effectiveness of SPRAT in reducing SPS among adolescents.

Methods and analysis This is a 6-month, cluster randomised clinical trial with two intervention arms (SPRAT vs usual school education). The study population will be composed of middle school students (aged 12-14 years) with their parents/guardians in Japan. SPRAT is expected to be a more powerful programme than PADOK as it reinforces the role of parent participation. The primary endpoint will be the change from baseline SPS scores to those obtained after 6 months. Between-group differences will be analysed following the intention-to-treat principle. Crude and multivariate adjusted effects will be examined using a general linear mixed-effects model for continuous variables and a logistic regression model for dichotomous variables. The sample size required was determined based on the information needed to detect a difference in the primary outcome with a significance level of $5 \%$ and power of $80 \%$ under the assumptions of 40 students per cluster (assuming the same sample size for each cluster), an effect size of 0.3 and an intraclass correlation coefficient of 0.02 . In total, participation by 28 schools ( 14 schools in each arm) (students: $n=1120$ ) will be needed. Ethics and dissemination This study was approved by the Medical Ethical Committee of Minami Kyushu University in 2017 (number 137). The findings will be disseminated widely through peer-reviewed publications and conference presentations.

Trial registration number UMIN000026715; Pre-results.

\section{INTRODUCTION}

Severe subjective psychosomatic symptoms (SPS) in adolescents are a major public health concern. ${ }^{1-5}$ Associations between
Strengths and limitations of this study

- SPRAT approach is an intervention to reinforce the role of parent participation in adolescents' healthy lifestyle modifications to reduce subjective psychosomatic symptoms and increase enjoyment of school life.

- This study is designed to evaluate changes in subjective psychosomatic symptoms scores according to the progression of SPRAT at 1 month, 3 months and 6 months after the baseline examination.

- Cluster randomised controlled trial is a powerful tool to evaluate school-based education directed towards changing adolescents' lifestyle.

- The study will be conducted in Japanese middle schools; therefore, generalisability is a limitation of the study design.

- The lack of blinding of the intervention to school teachers and registered dietitians is a limitation of the study design.

adolescents' lifestyles and SPS were shown, ${ }^{67}$ and several studies reported assessments of the effectiveness of interventions through targeting main lifestyle elements such as healthy dietary intake ${ }^{8-11}$ and physical activities $^{12-16}$ including daily routines. We had developed a school-based home-collaborative lifestyle education programme to improve poor SPS scores in adolescents Programme for adolescent of lifestyle education in Kumamoto (PADOK) based on our previous studies, ${ }^{817}$ and we proved the effectiveness of PADOK, though weakly. ${ }^{18}$ PADOK aimed to change adolescents' lifestyles in a non-compulsory way and to provide feedback on their dietary habits by assessments using the Food Frequency Questionnaire with 82 food items (FFQW82). ${ }^{19}$ The FFQW82 was developed to assess habitual dietary intake by evaluating nutritional intake at each meal. ${ }^{17} 19$ PADOK 


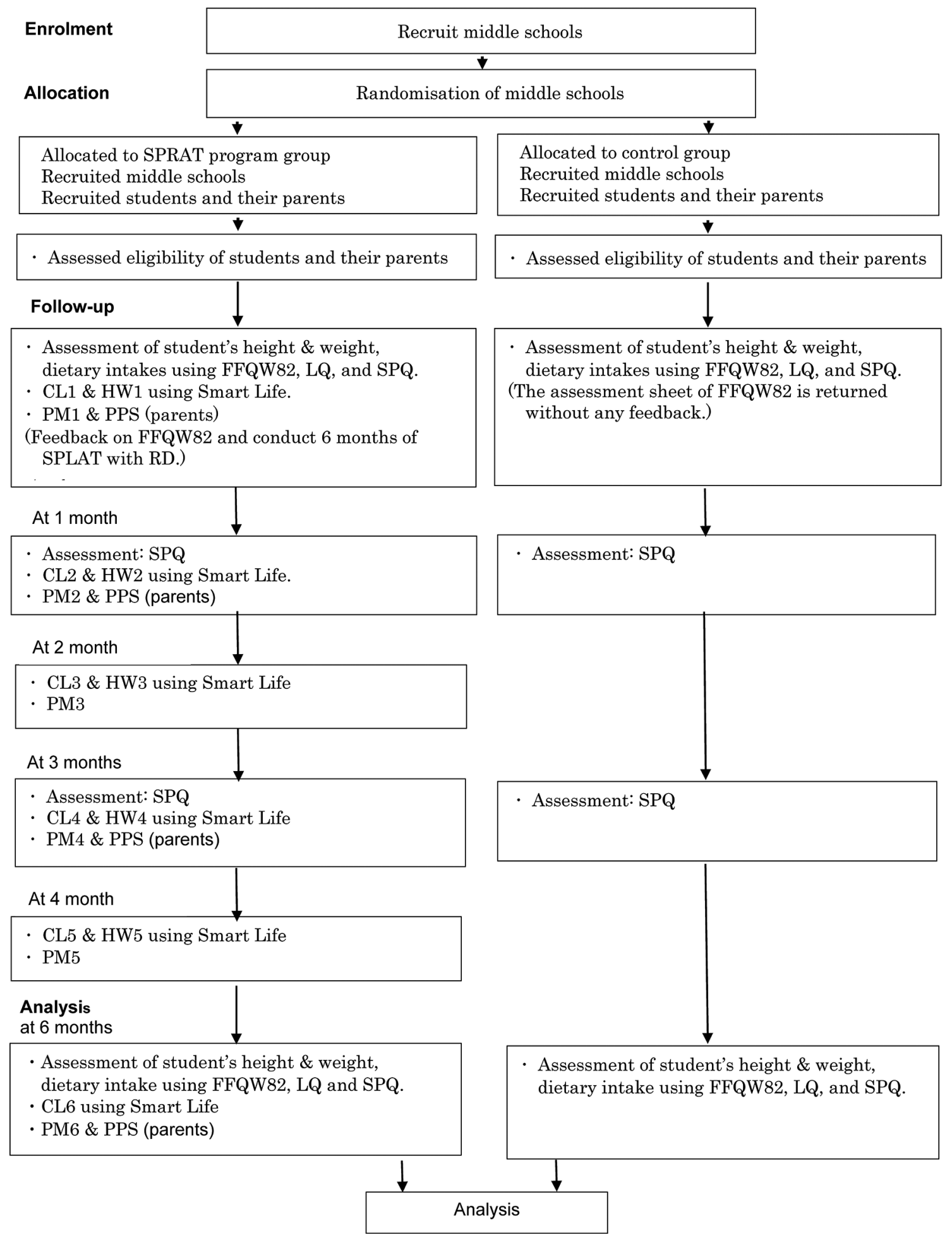

Figure 1 Flow diagram. CL, classroom lesson; FFQW82, Food Frequency Questionnaire with 82 food items; HW, homework; LQ, Lifestyle Questionnaire; Parents, parents/guardians; PM, parents' manual; PPS, parent-participation self-check sheet for parents/guardians; RD, registered dietitian; SPRAT, School-based lifestyle education involving parents for reducing subjective psychosomatic symptoms in Japanese adolescents; SPQ, Subjective Psychosomatic Symptoms Questionnaire.

had been assessed by a cluster randomised controlled trial (cRCT), and the results indicated that PADOK was effective in reducing SPS and promoting a healthy lifestyle for adolescents although the effect was statistically significant only in the crude model. ${ }^{18}$ Study findings indicated that SPS and chronic stress are prevalent among today's adolescents and that there is a critical need to develop effective and practical measures for minimising SPS and its potential influence among adolescents.

As for the mechanism of the linkage of dietary and lifestyle interventions with psychosomatic symptoms, several explanations have been proposed; the brain is 


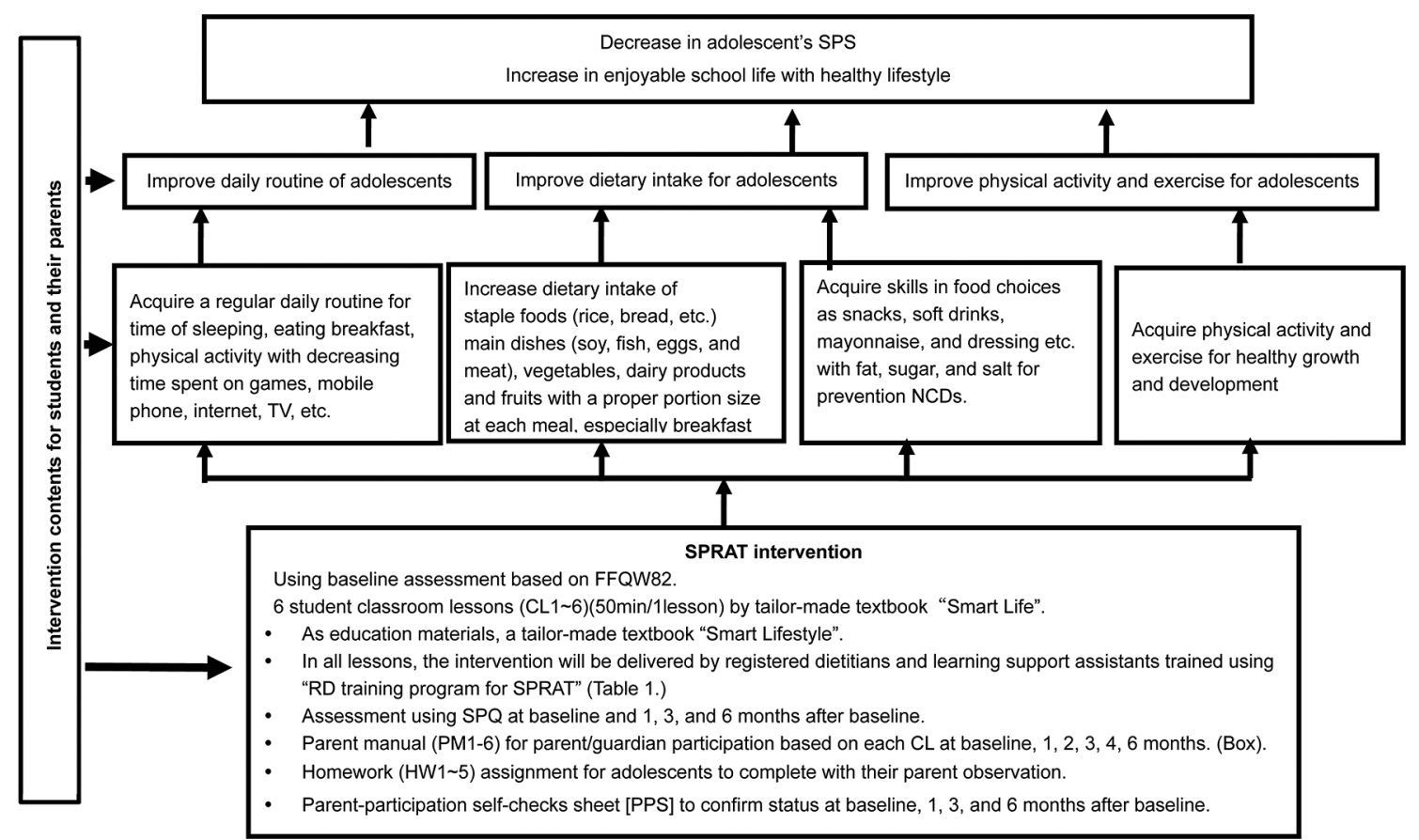

Figure 2 SPRAT intervention scheme. CL, classroom lesson; FFQW82, Food Frequency Questionnaire with 82 food items; HW, homework; LQ, Lifestyle Questionnaire; NCD, non-communicable disease; Parents, parents/guardians; PM, parents' manual; PPS, parent-participation self-check sheet for parents/guardians; RD, registered dietitian; SPRAT, School-based lifestyle education involving parents for reducing subjective psychosomatic symptoms in Japanese adolescents; SPQ, Subjective Psychosomatic Symptoms Questionnaire; SPS, subjective psychosomatic symptoms; TV, television.

entirely dependent on a continuous supply of glucose from the circulation since glucose is the major substrate for energy metabolism ${ }^{20}$; healthful breakfast consumption may improve cognitive function related to memory, test grades and school attendance, and breakfast as part of a healthful diet and lifestyle can positively impact children's health and well-being ${ }^{21}$; and the prefrontal cortex is activated in response to psychosocial stress and distinct prefrontal metabolic glucose patterns are linked to endocrine stress values as well as subjective ratings of task-related stress and controllability in addition to dispositional mood states. ${ }^{22}$

In the former home-collaborative lifestyle education intervention study of the PADOK, parents were not required to participate except for getting information on PADOK from four newsletters that were delivered or observations of their children's homework after informed consent. Furthermore, there was little consensus on the way to involve parents in treatment by home-collaborative lifestyle education. However, it may be important to reinforce the role of parent participation for reducing SPS in adolescents. Parents are in a unique position because adolescents' health behaviours are largely influenced by home-related factors, such as dietary intake and eating patterns at home, physical activity and a sedentary lifestyle. $^{23}$ For instance, in a meta-analysis review, some studies showed that parental involvement was associated with significantly better results in lifestyle interventions for youth who were overweight ${ }^{24}$; also, a systematic review showed that physically active parents were more likely to have physically active children, indicating parental influence. ${ }^{25}$ Another review concluded that although some effects of parental involvement were found in children's eating and physical activity behaviours, further studies on school-based interventions with parental components were needed. ${ }^{26}$ Comprehensive behavioural family lifestyle interventions demonstrated efficacy for improving weight outcomes in youths in a meta-analysis. ${ }^{27}$ Thus, previous research showed that parents play an important part in their adolescents' lifestyle education. Therefore, to establish a more effective programme, we have developed a school-based lifestyle education involving parents for reducing SPS in adolescents (SPRAT), an improved version of PADOK, which was used in the previous study. ${ }^{18}$

The aim of this study is to evaluate the effectiveness of SPRAT for reducing SPS in adolescents based on a cRCT.

\section{METHODS AND ANALYSIS}

\section{Study design and study setting}

The design of this study is an open-label, parallel group, school-based cRCT, with individual middle schools as the unit of allocation and individual participants as the unit of analysis. The study followed the Consolidated Standards of Reporting Trials (CONSORT) guidelines for the design. CONSORT flow diagram for the design of SPRAT study is shown in figure 1 .

The participant recruitment period will be from July 2018 to September 2018. The reason for requiring a long period between recruitment and randomisation is 


\section{Box 1 Scheme of the parent manuals}

1. Parent manuals will be provided.

2. Parent-participation self-check sheet will be completed at baseline, at 1,3 and 6 months.

3. Participation of parents/guardians will mainly include the following:

- Ensuring dietary intakes of staple foods (rice, breads, etc), main dishes (soy, fish, eggs and meat) and vegetables at breakfast.

Taking care to reduce fatty food consumption.

- Taking care to increase physical activity, such as at least $60 \mathrm{~min}$ of walking included going to school, activity and stretching by the manual in CL.

the time taken for coordination with school teachers and other stakeholders. The registration of participants will be completed from October 2018 to December 2019.

\begin{tabular}{|c|c|}
\hline Session & Contents \\
\hline 1 (1 hour) & $\begin{array}{l}\text { Introduction to SPRAT } \\
\text { a. background (PADOK, SPRAT) } \\
\text { b. aim } \\
\text { c. flow diagram (figure 1) } \\
\text { d. participants: middle school students and } \\
\text { their parents/guardians } \\
\text { e. assessments: students (height and weight, } \\
\text { FFQW82, LQ, SPQ) and parents/guardians } \\
\text { (PPS) } \\
\text { f. intervention: SPRAT intervention scheme } \\
\text { (figure 2) using 'Smart Life' and the parent } \\
\text { manual. }\end{array}$ \\
\hline 2 (2 hours) & $\begin{array}{l}\text { How to use the textbook 'Smart Life' and the } \\
\text { parent manual } \\
\text { a. Management of each CL (six CLs in total). } \\
\text { b. How to conduct baseline assessments: } \\
\text { students (height and weight, FFQW82, LQ, } \\
\text { SPQ) and parents/guardians (PPS). } \\
\text { c. How to conduct survey for parent/guardian: } \\
\text { PPS will be delivered to parents/guardians } \\
\text { by their child, and the completed PPS must } \\
\text { be returned to the classroom teacher the } \\
\text { next school day. } \\
\text { d. How to present the 'Smart Life'. } \\
\text { e. Homework. }\end{array}$ \\
\hline
\end{tabular}

3 (2 hours) Role play

a. opening session

b. CL1

c. final session

CL, classroom lesson; FFQW82, Food Frequency Questionnaire with 82 food items; LQ, Lifestyle Questionnaire; PADOK, Programme for adolescent of lifestyle education in Kumamoto; PPS, parent-participation self-check; RDTP, registered dietitian training programme; SPQ, Subjective Psychosomatic Symptoms Questionnaire; SPRAT, School-based lifestyle education involving parents for reducing subjective psychosomatic symptoms in Japanese adolescents; SPS, subjective psychosomatic symptoms.
The intervention follow-up period will be 6 months from randomisation (baseline). The primary endpoint will be a change from the baseline SPS score at 6 months. The SPS score will be obtained at baseline and at 1, 3 and 6 months based on a self-reported questionnaire (Subjective Psychosomatic Symptoms Questionnaire (SPQ)) for students.

\section{Participants and eligibility criteria}

The following procedure will be used to identify and enrol volunteer middle schools as participants:

i. Content of the trial will be explained and effort to obtain consent for schools' cooperation will be made at the Municipal Board of Education as well as the Principal Meetings in Kumamoto prefecture, Kumamoto city, Miyazaki prefecture and Miyazaki city.

ii. Requests for cooperation with the trial by telephone or a visit to all middle schools that can meet the requirement to make available a certain number of student participants (minimum of 40 students).

Criteria for student participation are as follows.

\section{Inclusion criteria}

i. Students in first and second grades (ages 12 to 14) and their parents/guardians from Miyazaki prefecture and Kumamoto prefecture middle schools that had agreed to cooperate with the study.

ii. Students and parents/guardians who freely consented to participate after demonstrating sufficient understanding of the study after a detailed explanation of its aims and procedures.

iii. Students and parents/guardians who agreed to cooperate with the trial and could comply with the procedures related to the trial according to the instructions of the researcher or the teacher.

\section{Exclusion criteria}

i. students who had mental disorders;

ii. students and parents/guardians of students who repeatedly refused to attend school in the past;

iii. students who were judged unsuitable for participation in the trial due to health reasons cited by the school nurse;

iv. students and parents/guardians who will be judged inappropriate to participate in the trial by teachers and researchers.

Thus, all eligible attendees of these schools in school years 1 and 2 (aged 12-14 years) and their parents/ guardians will be the participants in this study. SPRAT programme is focusing on cooperation with parents/ guardians because such cooperation is necessary for implementation of effective lifestyle improvements for adolescents. 


\section{Recruitment and allocation}

Subject recruitment

Written informed consent (online supplementary files 1 and 2) will be obtained from all participants, including both students and their parents/guardians. They will then provide evidence of consent to the school principals.

\section{Subject assignment}

Once the schools are recruited, we will randomly allocate them into SPRAT group or the usual school programme (control) group.

\section{Randomisation, allocation concealment and blinding}

Randomisation will be conducted using a permuted-block technique with the use of a randomisation list (random permutated blocks with block size 2). Random values will be obtained using personal computer (PC), and schools will be allocated manually by random numbers.

Due to the nature of the treatment, it is not possible to blind participants to the lifestyle education. However, data management team members, with the exception of the project coordinator and research assistants, will remain blinded to the group allocations until the code is opened after the last follow-up call is completed and the data are recorded.

The project coordinator and research assistants will not be blinded because they will be responsible for providing the education to participants. To minimise the risk of bias, strict protocols for follow-up assessment procedures (in Japanese) will be developed and research assistants will be trained to adhere to these protocols.

\section{Interventions}

SPRAT programme group (intervention group)

SPRAT is a lifestyle education programme involving parents to reduce SPS, increase enjoyment of school life and adopt healthier lifestyle factors including those related to diet and physical activity. The lifestyle education will be conducted based on the 'SPRAT intervention scheme' (figure 2) by registered dietitians. This study will be conducted according to the guidelines laid down in the Declaration of Helsinki.

SPRAT intervention will consist of six classroom lessons that will be provided once a month by trained registered dietitians. At each lesson, material for parents/guardians (parents manual) will be distributed in addition to five homework assignments for students to practice with their parents during the period between baseline and at 6 months. Lessons for the intervention will be conducted at a designated time by the junior high school (it depends on schools, namely some schools will use 'comprehensive learning', 'overall learning hour for the grade', 'home economics' and so on). In both intervention schools and control schools, students will take health classes as usually provided by schools. Parents/guardians in SPRAT group will be required to participate with regard to several issues according to the programme strategies (box 1 ).
The FFQW82 questionnaire, ${ }^{19}$ student's SPQ and student's Lifestyle Questionnaire (LQ) will be provided. For parents/guardians, parent-participation self-check sheets (PPS) will be provided to the intervention group. As teaching materials, 'Smart Life' will be delivered by registered dietitians to school teachers and to students to give to their parents/guardians.

SPRAT also aims to improve students' health behaviour and lifestyle habits. For instance, increasing enjoyment of school life and health; decreasing time spent on computer games, mobile phones, internet and watching television; sleeping at regular hours each day and eating breakfast. The programme also encourages students to increase their intake of staple foods (rice, bread, etc), main dishes (soy, fish, eggs and meat) and vegetables, particularly at breakfast and helps students to develop skills in food selection by delivering advice and education. For example, they are urged not to consume high-fat foods such as mayonnaise and dressings or highsugar drinks and to increase their daily physical activity and exercise.

\section{Usual care (control group)}

Students in the usual school programme (control) will participate in health education classes provided by the school according to the usual curriculum. The usual care will be composed of any existing health curriculum about diet and/or exercise that is routinely taught at each participating school. Dietary assessment using the FFQW82 will be conducted additionally by the teacher.

All of the questionnaires surveys will be administered by registered dietitians who will visit the junior high school. The registered dietitians will be trained beforehand according to the manual. They will respond to all questions from the students.

Distribution/collection of questionnaires to parents/ guardians will be done through teachers in the junior high schools.

\section{Registered dietitians' training programme for SPRAT}

The success of SPRAT will be to some extent dependent on the skills of the dietitians. Training for the registered dietitians and learning support assistants will be provided based on the 'registered dietitian training programme' for SPRAT by the trial leader, who will be a registered dietitian (table 1).

The training will take place over a 5-hour period at the study management centre in Miyazaki and Kumamoto. During the training day, the rationale for the intervention will be explained, and each lesson and homework activity will be discussed interactively.

\section{Study hypothesis}

The hypothesis underlying this study is that students who participated in SPRAT group will show a greater decrease in their mean SPS score than students who participated in the control group after 6 months. 


\section{Outcome measures and background variables}

Primary outcome

The primary outcome will be the SPS score measured by responses to the questionnaire. The SPS questionnaire (SPQ) consists of questions on nine symptoms (fatigue, headache, lassitude, irritation, lack of concentration, lack of motivation, poor ability to wake up in the morning, upset stomach and stiff shoulder). The response categories for the frequency of experiencing each symptom were ' $0=$ never', ' $1=$ =rarely', ' $2=$ sometimes', ' $3=$ often', and '4=always.' Using a Likert scale, we will calculate the SPS score (0-36 points) as the sum of the category values for nine items. Primary endpoint will be the difference between the two groups in relation to changes in SPS scores from baseline to 6 months $( \pm 1$ month). The validity and reliability of the SPS score were shown elsewhere. ${ }^{18}$

\section{Secondary outcomes}

Secondary outcomes will be lifestyle factors (sleeping habits, eating breakfast, consuming fatty snacks or meals and soft drinks, time spent on computer games, etc, enjoyable school life and physical activity) and dietary intake assessed by the FFQW $82^{19}$ as well as body mass index (BMI). The FFQW82 consists of a list of 82 foods with colour illustrations showing portion sizes, and the dietary habits of respondents for the previous 1 month can be calculated for 1 day and each meal (breakfast, lunch and dinner) by food groups. The validity and reliability of the FFQW82 were previously established for adolescents. ${ }^{17}$ Details of the FFQW82 and the assessment method were reported elsewhere. ${ }^{28}$ Also, the Japanese version of the dietary assessment software using FFQW82 can be downloaded from the publisher's website. The secondary endpoints will be related to changes in values pertaining to the secondary outcomes.

\section{Background variables}

Information on student's background (sex, age, height, weight) and parent's background (sex, age, relationship with child (father, mother, guardian) will be obtained from the student's LQ and the PPS at baseline and 6 months.

In addition, we will examine the characteristics of the schools regarding their type (private vs public school), location (rural vs urban area) and academic performance (high-ranking vs other).

\section{Statistical analysis}

\section{Sample size}

The sample size required for the study was determined based on the information needed to detect a difference in the primary outcome with a significance level of $5 \%$ and power of $80 \%$, under the assumptions of 40 students per cluster (assuming the same sample size for each cluster), an effect size (for severe SPS) of 0.3 and an intraclass correlation coefficient of 0.02 . The effect size was estimated from our experience in a former study. ${ }^{29}$ In total, participation by 28 schools ( 14 schools in each arm) (students: $\mathrm{n}=1120$ ) will be needed.

\section{Statistical analysis}

We will use descriptive statistics to assess the balance between the trial arms at baseline. To ensure that cluster randomisation is successful, the significance of differences between the intervention and control groups will be examined using the $\chi^{2}$ test and t-test. The primary endpoint is the change from the baseline SPS score to that at 6 months. The difference between the two groups will be examined based on an intention-to-treat (ITT) principle with the full analysis set and imputation of missing data will be performed using the last observation carried forward method (ITT/LOCF) and a multiple imputation method (ITT/multiple imputation) using chained equations under the assumption of missing at random. ${ }^{30} \mathrm{~A}$ linear random-effects mixed model employing the maximum likelihood method will be used for the analyses. A general linear random-effects mixed model employing the restricted maximum likelihood method will be used for the analysis of continuous variables. Outcome measures will be used to examine the effects of the intervention by a crude model (model 1), a model adjusted for baseline values (model 2) and a multivariate-adjusted model (adjusted for baseline, sex, age and BMI) (model 3).

Secondary analyses will be conducted for the secondary outcomes the same as for the primary analyses using the ITT/LOCF approach. Sensitivity analyses will be conducted for the primary and secondary outcomes using a per protocol set identified from the complete data set following the criteria determined a priori including analyses for the SPS score. As for the dichotomous secondary outcomes, a generalised linear random effects mixed model (logistic model) will be used for the analysis and associations will be shown as an OR and its 95\% CI.

All tests for significance will be conducted using a two-sided approach with a 5\% significance level. All statistical analyses will be performed using SAS V.9.4 for Windows (SAS Institute).

\section{Time frame of the study}

Middle schools will be registered as study participants from April to December in 2018, and all students and their parents/guardians will be registered from April to December in 2019. The baseline measurements will be started in April 2018. Data on the effect evaluation will be available in 2019. The time frame of the interventions is shown in figure 1.

\section{Data management}

Personal information obtained in this study will be coded and anonymised. All data and documents related to the present study will be managed securely for 5 years from the completion of the study by the Minami Kyushu University. All materials will be disposed of by shredding, and sufficient care will be taken to ensure that names of 
individuals in the agreements cannot be identified. Data will be encoded directly to the standalone PC and saved. The security of databases and networks will be regularly updated and backups made. The completed questionnaires and the Universal Serial Bus storage memories will be stored in a dedicated cabinet with a key.

\section{Conditions for discontinuation of participation in this study and actions in that event}

1. If a participant withdraws consent for participation in the study.

2. If a school teacher determines that the continuation of the lifestyle education is not preferable for student.

3. If a student's teacher determines that discontinuing the study is appropriate for the student for other reasons.

4. If the study protocol cannot be completely followed.

\section{Monitoring}

Information on when the study begins, conduct of the study (sample size), ethical considerations, occurrence of detrimental or adverse events, results of the study and registration of the study with a public database will be submitted to our ethics committee in an annual interim report. A report will also be submitted to the ethics committee on the conclusion of the study and when the final results are presented.

\section{Protocol amendments}

If any amendments of the protocol are made, the Ethics Committee may be notified as necessary.

\section{Follow-up of adverse events}

This study involves implementation of a lifestyle programme to improve the lifestyles of participants and for participants to receive education from a registered dietitian in a school class. There is little likelihood of any health hazards. If any serious adverse events do occur, they will be reported in line with the standard operating procedure on Reporting Serious Adverse Events in Clinical Research.

\section{Ethics and dissemination}

The study is implemented in accordance with the Declaration of Helsinki and the ethical guidelines for medical research covering humans.

The present study is an open-label study. Only the students will be blinded because the students' teachers and registered dietitians in the school education settings cannot be blinded. All participants are given an ID and data are anonymised. Thus, no information identifying specific students is stored alongside study data. It is considered that the possibility of harm to the students is minimal because the examinations and education will be performed in the setting of usual school education. However, due consideration will be given to telling the participants that if they want to discontinue because of the psychological burden, they can do so at any time, and the required procedure for withdrawal will be followed.
The same applies to collaborating parents/guardians. The results of the study will be presented in conferences and published in international journals. Also, we will hold seminar/lecture to generalise the study results. Furthermore, we encourage national and relevant organisations to use the findings obtained.

\section{DISCUSSION}

Severe SPS in adolescents are a major public health concern, and an effective lifestyle education programme to improve SPS and promote a healthy lifestyle for adolescents is warranted. The findings of the study will correspond to the critical need to develop effective practical measures for minimising SPS and its potential influence among adolescents. The inclusion of parent-participation support may help to obtain favourable effects. ${ }^{31}$ Thus, it is important that an interdisciplinary team is established and horizontal cooperation between researchers and school teachers is achieved in approaching the research question and study design in addition to parent-participation support. SPRAT programme is the first study design for effecting school-based lifestyle education involving parents for reducing SPS in middle school students in Japan. The trial is designed to test our hypothesis that students who complete SPRAT programme will have improved their SPS score, increase enjoyment of school life and adopt healthier lifestyle factors including those related to diet and physical activity. In this study, the SPS score for students will be observed to monitor changes from baseline to 6 months.

The intervention by registered dietitians should result in success from the point of view of accuracy and homogenisation based on completion of the registered dietitian training programme for SPRAT. In this study, the tailormade textbook will be a useful tool for parent participation lifestyle education as well as for classroom sessions, homework and communication among the students, their parents/guardians and the registered dietitians.

Contributors JW, MW, KY, MA and TT were responsible for designing the research questions and drafted the study protocol. JW, MW, KY and AN will be responsible for data management. JW and MW will be responsible for management of dietary education. KY, TT and AN will be responsible for statistical analyses. JW, MW and $\mathrm{KY}$ wrote the first draft of this paper. All authors read and approved the final manuscript.

Funding This study is financially supported by Academic Research Community Contribution Miyazaki City Grants in 2017.

Disclaimer The funding source had no role in the design of this study and will have no role during its execution, analyses, interpretation of the data or in any decision to submit results.

Competing interests None declared.

Patient consent Obtained.

Ethics approval This study was approved by the Medical Ethical Committee of Minami Kyushu University in 2017 (number 137).

Provenance and peer review Not commissioned; externally peer reviewed.

Open Access This is an Open Access article distributed in accordance with the Creative Commons Attribution Non Commercial (CC BY-NC 4.0) license, which permits others to distribute, remix, adapt, build upon this work non-commercially, 
and license their derivative works on different terms, provided the original work is properly cited and the use is non-commercial. See: http://creativecommons.org/ licenses/by-nc/4.0/

(C) Article author(s) (or their employer(s) unless otherwise stated in the text of the article) 2018. All rights reserved. No commercial use is permitted unless otherwise expressly granted.

\section{REFERENCES}

1. Kieling $\mathrm{C}$, Baker-Henningham $\mathrm{H}$, Belfer $\mathrm{M}$, et al. Child and adolescent mental health worldwide: evidence for action. Lancet 2011;378:1515-25.

2. Cabinet Office (Japan). Heisei 26, Version children and youth white paper (Central Edition). 2016. http://www8.cao.go.jp/youth/ whitepaper/h26honpen/ (accessed 29 Jul 2017).

3. Crawley EM, Emond AM, Sterne JA. Unidentified chronic fatigue syndrome/myalgic encephalomyelitis (CFS/ME) is a major cause of school absence: surveillance outcomes from school-based clinics. BMJ Open 2011;1:e000252.

4. Tanaka H, Terashima S, Borres MP, et al. Psychosomatic problems and countermeasures in Japanese children and adolescents. Biopsychosoc Med 2012;6:6.

5. Langford R, Bonell CP, Jones $\mathrm{HE}$, et al. The WHO health promoting school framework for improving the health and well-being of students and their academic achievement. Cochrane Database Syst Rev 2014;4:CD008958.

6. Melnyk BM, Small L, Morrison-Beedy D, et al. Mental health correlates of healthy lifestyle attitudes, beliefs, choices, and behaviors in overweight adolescents. J Pediatr Health Care 2006;20:401-6.

7. Isshiki Y, Morimoto K. Lifestyles and psychosomatic symptoms among elementary school students and junior high school students. Environ Health Prev Med 2004;9:95-102.

8. Yamaoka K, Watanabe M, Hida E, et al. Impact of group-based dietary education on the dietary habits of female adolescents: a cluster randomized trial. Public Health Nutr 2011;14:702-8.

9. Caballero B, Clay T, Davis SM, et al. Pathways: a school-based, randomized controlled trial for the prevention of obesity in American Indian schoolchildren. Am J Clin Nutr 2003;78:1030-8.

10. James J, Thomas P, Cavan D, et al. Preventing childhood obesity by reducing consumption of carbonated drinks: cluster randomised controlled trial. BMJ 2004;328:1237.

11. Grydeland M, Bjelland M, Anderssen SA, et al. Effects of a 20-month cluster randomised controlled school-based intervention trial on BMI of school-aged boys and girls: the HEIA study. Br J Sports Med 2014;48:768-73.

12. Metcalf B, Henley W, Wilkin T. Effectiveness of intervention on physical activity of children: systematic review and meta-analysis of controlled trials with objectively measured outcomes (EarlyBird 54). BMJ 2012;345:e5888.

13. Kipping RR, Howe LD, Jago R, et al. Effect of intervention aimed at increasing physical activity, reducing sedentary behaviour, and increasing fruit and vegetable consumption in children: active for Life Year 5 (AFLY5) school based cluster randomised controlled trial. BMJ 2014;348:g3256.

14. Sutherland R, Campbell E, Lubans DR, et al. 'Physical activity 4 everyone' school-based intervention to prevent decline in adolescent physical activity levels: 12 month (mid-intervention) report on a cluster randomised trial. Br J Sports Med 2016;50:488-95.

15. Murillo Pardo B, García Bengoechea E, Generelo Lanaspa E, et al. Promising school-based strategies and intervention guidelines to increase physical activity of adolescents. Health Educ Res 2013;28:523-38.

16. De Bourdeaudhuij I, Van Cauwenberghe E, Spittaels H, et al. Schoolbased interventions promoting both physical activity and healthy eating in Europe: a systematic review within the HOPE project. Obes Rev 2011;12:205-16.

17. Watanabe M, Yamaoka K, Yokotsuka M, et al. Validity and reproducibility of the FFQ (FFQW82) for dietary assessment in female adolescents. Public Health Nutr 2011;14:297-305.

18. Watanabe J, Watanabe M, Yamaoka K, et al. Effect of school-based home-collaborative lifestyle education on reducing subjective psychosomatic symptoms in adolescents: a cluster randomised controlled trial. PLoS One 2016;11:e0165285.

19. Adachi M, Watanabe M, Yamaoka K, et al. [Validity and reproducibility of a food frequency questionnaire with 82 -food items (FFQW82) for nutrition education]. Nihon Koshu Eisei Zasshi 2010;57:475-85.

20. Duelli R, Kuschinsky W. Brain glucose transporters: relationship to local energy demand. News Physiol Sci 2001;16:71-6.

21. Rampersaud GC, Pereira MA, Girard BL, et al. Breakfast habits, nutritional status, body weight, and academic performance in children and adolescents. J Am Diet Assoc 2005;105:743-60.

22. Kern S, Oakes TR, Stone CK, et al. Glucose metabolic changes in the prefrontal cortex are associated with HPA axis response to a psychosocial stressor. Psychoneuroendocrinology 2008;33:517-29.

23. Patino-Fernandez AM, Hernandez J, Villa M, et al. School-based health promotion intervention: parent and school staff perspectives. J Sch Health 2013;83:763-70.

24. Kitzmann KM, Dalton WT, Stanley CM, et al. Lifestyle interventions for youth who are overweight: a meta-analytic review. Health Psycho 2010;29:91-101.

25. Edwardson CL, Gorely T. Parental influences on different types and intensities of physical activity in youth: a systematic review. Psychol Sport Exerc 2010;11:522-35.

26. Van Lippevelde W, Verloigne M, De Bourdeaudhuij I, et al. Does parental involvement make a difference in school-based nutrition and physical activity interventions? A systematic review of randomized controlled trials. Int J Public Health 2012;57:673-8.

27. Janicke DM, Steele RG, Gayes LA, et al. Systematic review and meta-analysis of comprehensive behavioral family lifestyle interventions addressing pediatric obesity. J Pediatr Psychol 2014;39:809-25

28. Yamaoka K, Adachi M, Watanabe M, et al. Raifusutairu kaizen no jissen to hyouka (in Japanese) (Practice and evaluation of lifestyle modification). Tokyo: Asakura Shoten Co., Ltd. Press, 2015. (accessed 29 Jul 2017).

29. Watanabe J, Watanabe M, Yamaoka K, et al. [The association between subjective symptoms and lifestyle habits among junior high school students a cross-sectional survey in Kumamoto, Japan]. Nihon Koshu Eisei Zasshi 2016;63:113-25.

30. White IR, Royston P, Wood AM. Multiple imputation using chained equations: issues and guidance for practice. Stat Med 2011;30:377-99.

31. Community Preventive Services Task Force. Improving adolescent health through interventions targeted to parents and other caregivers: a recommendation. Am J Prev Med 2012;42:327-8. 